\title{
Experimental Approaches to Improve Thermal Stability of Organic Phase Change Properties
}

\author{
Amin Al Irobaidi \\ Faculty of Engineering, Materials Department, AlBalq'a Applied University (BAU), Salt, Jordan \\ Email: arobaidi@yahoo.de
}

How to cite this paper: Al Irobaidi, A. (2018) Experimental Approaches to Improve Thermal Stability of Organic Phase Change Properties. Journal of Materials Science and Chemical Engineering, 6, 125-135.

https://doi.org/10.4236/msce.2018.67014

Received: June 9, 2018

Accepted: July 15, 2018

Published: July 18, 2018

Copyright $\odot 2018$ by author and Scientific Research Publishing Inc. This work is licensed under the Creative Commons Attribution International License (CC BY 4.0).

http://creativecommons.org/licenses/by/4.0/

\begin{abstract}
This study investigates the thermal behavior of Polyolefin containing Paraffin and Nano Hydrated aluminum silicate $\mathrm{Al}_{2} \mathrm{Si}_{2} \mathrm{O}_{5}(\mathrm{OH})_{4}$ (Kaolin) particles to enhance store energy at ambient temperature. The hybrid Nano composite is based on polyolefin PE as a matrix, whereby paraffin wax and Kaolin were hot blended at varying concentrations. In addition Carbon Nanotube (CNTs) was added in different relative low concentrations to improve the thermal transition among the polymer matrix, since polymer domains are considered as isolator. The composite was prepared by melt mixing using a Brabender Plasrograph and a Two Role Mill. Thermal properties of the composite were determined using DSC and Melt flow Index. Because TES materials are subjected to melting and freezing during life time, multiple extrusion tests to simulate the degradation process of the composite were carried out. FTIR was applied to determine the degradation effect and investigate microstructure changes of the composite. The results obtained demonstrate that the blend shows a tendency to be thermally active at low temperatures. DSC tests evidenced a decrease in melt temperature as a result of increasing Kaolin content and some changes in the latent heat of the compound.
\end{abstract}

\section{Keywords}

Phase Change Materials, Polymer Composites, Nano Kaolin, TES, Carbon Nanotubes, PE-Wax

\section{Introduction}

Thermal energy storage (TES) by solar power has become a popular research topic in recent years. Phase Change Materials (PCMs) typically used in thermal applications is usually grouped by their chemical makeup ("organic" or "inorganic"), with the most common being organic [1] [2] [3] [4] [5]. 
The continuous and increasing efforts to utilize alternative and sun energies to reduce and replace hydrocarbon fuel utilization, especially after the climb in fuel prices are the main driving forces behind efforts into more effectively utilize various sources of renewable energy. In many parts of the world, direct solar radiation is considered to be one of the most prospective sources of energy. However, the large-scale utilization of this form of energy is possible only if new materials with large capacity of heat of fusion is achieved and the effective technology for its storage can be developed with acceptable capital and running costs [1]. One of prospective techniques of storing solar energy is the application of phase change materials (PCMs). This paper looks at the current state of research in this particular field, with the main focus being to develop composite with increased storage capacities.

The utilization of latent heat storage capability of phase change materials is one of the keys to an efficient way to store thermal energy. There are large numbers of PCMs that melt and solidify at a wide range of temperatures, making them attractive to a number of applications. PCMs typically used in thermal applications are usually grouped by their chemical makeup ("organic" or "inorganic"), with the most common being organic [6]-[12].

Phase change material was broadly investigated to develop efficient materials for energy storage by many authors. Polymers and other organic compound and salt minerals etc... were investigated and reported in scientific Journals [4]-[10]. Phase change material based on salt hydrate for commercial application for building and other sectors for energy saving are commercialized [13] [14] [15].

Some Polymer phase change materials are currently used for encapsulating of PCM; whereby a combination of Polyethylene's and paraffin is still under investigation. Different organic PCMs are available in broad range of Temperature; depend on type of application.

Different thermoplastics and rubber composites containing Nano carbon tube as well as special additives to improve efficient use of PCMs will result in changing its storage behavior [12]-[19].

Latent heat TES systems have recently attracted many researchers, while these systems are considered to have a high energy storage density at constant temperature corresponding to the transition temperature of the phase change material (PCM) [19]-[23].

Researchers have to alter PCM properties in the desired way to increase its ability to store latent heat. The gain can be used to develop new system for all kind of applications. As long as the PCM phase change temperature is within the desired product temperature range [9] [10] [11]. In this paper an attempt was undertaken to add Kaolin; Carbon Nano Tube (CNT) nanoparticles; to a relatively low molecular weight Polyethylene containing vinyl acetate with a melting temperature around $75^{\circ} \mathrm{C}$. Because of the impact on day and night on solar thermal energy storage, thus, the development of efficient energy storage mate- 
rials will directly influence the utilization efficiency of solar thermal energy storage. However; it is difficult to create heat storage materials that have a large heat storage density of relative low temperatures $\left(<100^{\circ} \mathrm{C}\right)$. Efforts have yet uncovered materials with sufficient functionality of polymeric phase change materials. This research project is an attempt to find or develop new thermoplastic materials hybrid composites that can be used as promising new thermal energy storage materials. Melting point and latent are a significant parameter to evaluate the ability of using such polymer for heat storage applications. DSC thermo grams can accurately measure enthalpy (latent heat), melting and freezing point of the materials. In addition DSC thermograms are used to measure crystallinity of polymers that are of significant for the heat storage behavior. Other test methods including FTIR and SEM are also used to characterize micro structure and material properties. Meanwhile an ASTM standard testing is developed to evaluate such materials. Focal point in a promising development is the cost of such materials. Commodity items in different applications are considered to be still expensive.

A theoretical model to predict the phase change behavior of the material with temperature-dependent specific heat can be used to predict the phase change processes in the most common PCM materials.

The amount of thermal energy stored in the form of sensible heat can be calculated by using the heat capacity equation:

$$
Q=m * C p \int_{T 1}^{T 2} \mathrm{~d} T
$$

Heat capacity $=$ mass $\times$ specific heat $\times$ change in temperature

$Q=$ heat capacity, $\mathrm{J}$

$m=$ mass, $\mathrm{g}$

$c=$ specific heat of object, $\left(\mathrm{J} / \mathrm{g}-{ }^{\circ} \mathrm{C}\right)$

$\Delta T=$ change in temperature, ${ }^{\circ} \mathrm{C}$

Since the polymer conductivity is low; CNTs were added to improve heat transfer. Considering multi component the one-dimensional heat transport equation for such a case is as follows:

$$
\frac{\partial}{\partial t}(\rho h)=\frac{\partial}{\partial x}\left[\lambda \frac{\partial T}{\partial x}\right]
$$

where $\rho$ is the material density, $\lambda$ is the thermal conductivity, and $h$ is enthalpy per unit mass.

The enthalpy derivative over the temperature derivative (with consideration of constant Pressure) represents the effective heat capacity $\left(C_{e f f}\right)$, with the phase change energy as one of the components:

$$
C_{e f f}=\frac{\partial h}{\partial T}
$$

For most PCMs, variations on enthalpy associated with temperature depend to some extent on the direction of the process considered, and tend to differ from melting and solidification (Gunther et al. 2009). Transient characteristics 
of PCM-enhanced products depend on the PCM content and the quality of the PCM carrier. Usually, a smaller portion of the heat storage capacity (depending on the temperature difference) consists of sensible heat; a larger capacity portion represents heat of the phase transition. Effective heat capacity, $C_{\text {eff }}$ for a material consisting of a blend of the material carrier and PCM can be expressed as follows:

$$
c_{\text {eff }}=(1-\alpha) c a r r+\alpha_{\text {ceff }}{ }_{P C M},
$$

where $\alpha$ denotes the percentage of PCM, $\mathrm{C}_{\text {eff }}$ is the specific heat of a carrier material without PCM, and $\mathrm{C}_{\text {eff }}$ is the effective heat capacity of the PCM.

\section{Experimental}

\subsection{Materials}

Low molecular weight PE pellets provided by DuPont Elvax ${ }^{\circledR} 265$ with a melting point $(\mathrm{Tm})$ around $75^{\circ} \mathrm{C}$ and heat fusion of $44 \mathrm{~J} / \mathrm{g}$ were used. Elvax are modified ethylene vinyl acetate polymers used as adhesive resin for many applications. They are available in pellet form for use in conventional extrusion and extrusion equipment designed to process polyethylene (PE) resins. This PE resin was chosen because of its low softening points and excellent specific heat values that make it useful to be modified for heat storage applications. As per DuPont Data sheet the Melt flow Index (MFI) is equal to $3 \mathrm{~g} / 10 \mathrm{~min}$ at $\left(190^{\circ} \mathrm{C} / 2.16 \mathrm{~kg}\right)$. Nano Kaolin in powder forms, particle size diameter in the range (110 - 140 Nano) was used in different concentration. XRF technique is very common characterization method used to determine kaolin composition and it was applied to identify the mineralogical composition of the kaolin. The chemical composition analysis is presented in Table 1. Paraffin, with a melting point of $10^{\circ} \mathrm{C}$ was provided by Rubitherm Technologies (Germany). Multi walled nanotubes (MWCNTs) with a mean diameter of $9.5 \mathrm{~nm}$ produced by Nanocyl SA (Belgium where added at a fixed low concentration $0.25 \%$ to enhance thermal percolation of the TES compound. In Table 2 Percentage of LDPE, CTN; Kaolin and Paraffin Wax in each specimen are shown.

\subsection{Sample Preparation}

All Ingredients PE, paraffin, and MWCNTs as listed in Table 3, were melt compounded in a Brabender Plastograph with sigma-rotating rotors. After various preliminary attempts, all the blends were prepared at constant processing conditions. Temperature $\left(75^{\circ} \mathrm{C}\right)$, rotor speed applied $(25 \mathrm{rpm})$ and a mixing

Table 1. Mineralogical composition (XRF) of Saudi Kaolin.

\begin{tabular}{cccccccc}
\hline \multicolumn{7}{c}{ Saudi Kaolin Chemical Composition $($ Weight $)$} \\
\hline $\mathrm{SIO}_{2}$ & $\mathrm{AL}_{2} \mathrm{O}_{3}$ & $\mathrm{FE}_{2} \mathrm{O}_{3}$ & $\mathrm{MgO}$ & $\mathrm{CaO}$ & $\mathrm{K}_{2} \mathrm{O}$ & $\mathrm{TIO}_{2}$ & $\mathrm{P}_{2} \mathrm{O}_{5}$ \\
\hline 57.4 & 37.7 & 0.89 & 0.69 & 0.54 & 1.8 & 0.66 & 0.32
\end{tabular}


Table 2. Indicates the concentration of each component of the prepared samples.

\begin{tabular}{ccccc}
\hline Sample No. & LDPE & Paraffin Wax & CNT & Kaolin \\
\hline 1 & $100 \%$ & - & - & - \\
2 & - & $100 \%$ & - & - \\
3 & $85 \%$ & $10 \%$ & - & $10 \%$ \\
4 & $75 \%$ & $15 \%$ & - & $15 \%$ \\
5 & $65 \%$ & $15 \%$ & - & $25 \%-$ \\
6 & $55 \%$ & $45 \%$ & - & - \\
7 & $80 \%$ & $19.75 \%$ & $0.25 \%$ & - \\
8 & $70 \%$ & $29.75 \%$ & $0.25 \%-$ & - \\
9 & $60 \%$ & $39.75 \%$ & $0.25 \%-$ & - \\
10 & $50 \%$ & $49.75 \%$ & $0.25 \%-$ & $5 \%$ \\
11 & $80 \%$ & $14.75 \%$ & $0.25 \%-$ & $10 \%$ \\
12 & $70 \%$ & $19.75 \%$ & $0.25 \%-$ & $15 \%$ \\
13 & $60 \%$ & $24.75 \%$ & $0.25 \%-$ & $25 \%$ \\
14 & $50 \%$ & $24.75 \%$ & $0.25 \%-$ & \\
\hline
\end{tabular}

Table 3. Indicates phase transition parameters $T_{m}$ and the heat of fusion $\Delta H_{c}$ for the 14 samples as determined from DSC thermograms in Figures 1-3.

\begin{tabular}{ccccccccccccccc}
\hline Sample No. & $\mathbf{1}$ & $\mathbf{2}$ & $\mathbf{3}$ & $\mathbf{4}$ & $\mathbf{5}$ & $\mathbf{6}$ & $\mathbf{7}$ & $\mathbf{8}$ & $\mathbf{9}$ & $\mathbf{1 0}$ & $\mathbf{1 1}$ & $\mathbf{1 2}$ & $\mathbf{1 3}$ & $\mathbf{1 4}$ \\
\hline $\begin{array}{c}\text { Melting } \\
\text { point } \mathrm{T}_{\mathrm{M}}{ }^{\circ} \mathrm{C}\end{array}$ & 75.9 & 74.4 & 75.9 & 73.4 & 72.3 & 66.8 & 65.9 & 65.4 & 65.2 & 62.3 & 71.5 & 74.2 & 70 & 49.3 \\
$\begin{array}{c}\text { Enthalpy } \\
\Delta \mathrm{H}_{\mathrm{c}} \mathrm{kJ} / \mathrm{g}\end{array}$ & 71.31 & 154.52 & 131.6 & 138.8 & 143.9 & 148.4 & 126.5 & 124.3 & 131.6 & 143.9 & 140.5 & 126.6 & 135.3 & 143.9 \\
\hline
\end{tabular}

time (5 minutes. Sample were taken in a molten stage and inserted rapidly into a heated two roll mill to produce sheets in $1 \mathrm{~mm}$ thickness. The sheets were grinded and on a Pelletizer extruder, pellets were produced. Those pellets were later subjected to multiple extrusions.

\subsection{Experimental Technique}

\subsubsection{Thermal Properties}

Differential scanning calorimetric (DSC) was carried out with a Perkin Elmer DSC machine under a nitrogen flow of $150 \mathrm{~mL} / \mathrm{min}$. Heating/cooling rate was set at $10^{\circ} \mathrm{C} / \mathrm{min}$ for all the measurements, starting from -10 up to $100^{\circ} \mathrm{C}$. In this way, melting/crystallization temperatures $\left(\mathrm{T}_{\mathrm{m}}, \mathrm{T}_{\mathrm{c}}\right)$ and melting/crystallization enthalpy $\left(\Delta \mathrm{H}_{\mathrm{m}}, \Delta \mathrm{H}_{\mathrm{c}}\right)$ of paraffin and of the compound were measured.

Figures 1-3 presents the DSC first heating thermograms of the samples 1 - 14 as stated in Table 1 . The area under the curve of melting peaks in the thermograms is proportional to the enthalpy of the prepared compound. The first peak at the temperature range $15^{\circ} \mathrm{C}$ to $40^{\circ} \mathrm{C}$ in the thermograms is related to the paraffin wax; whereby the melting of the Polyethylene's is seen in the second peak, 


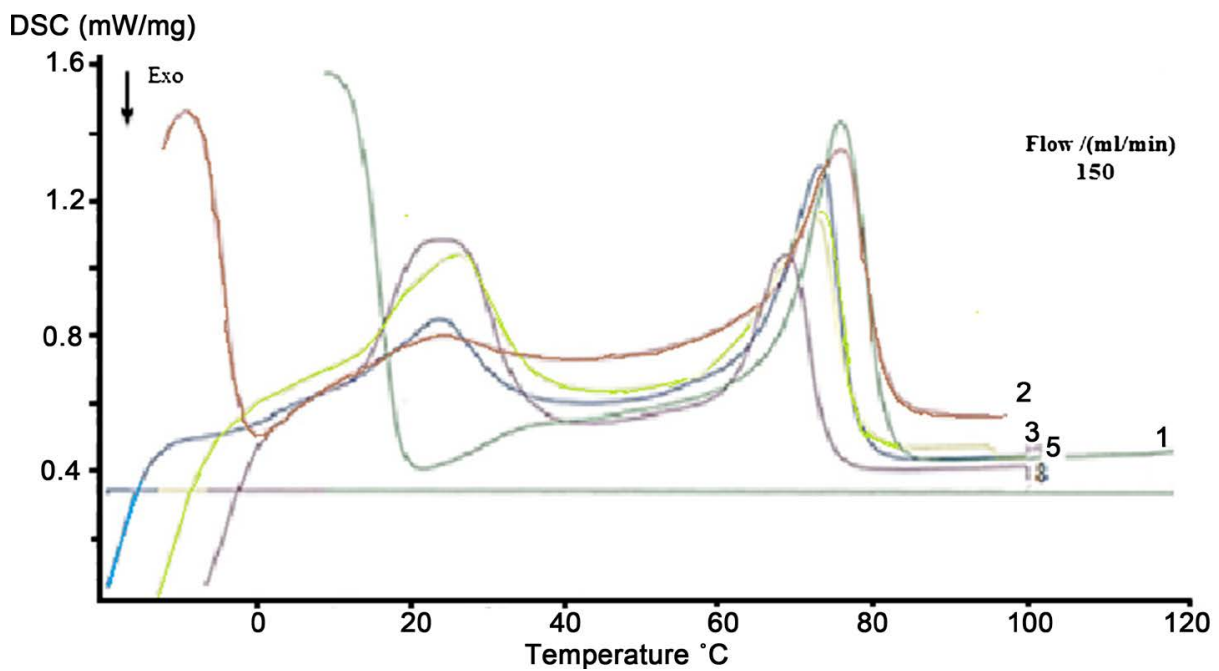

Figure 1. DSC Thermograms for the samples No's 1, 2, 3, 5, 8 as listed in Table 1.

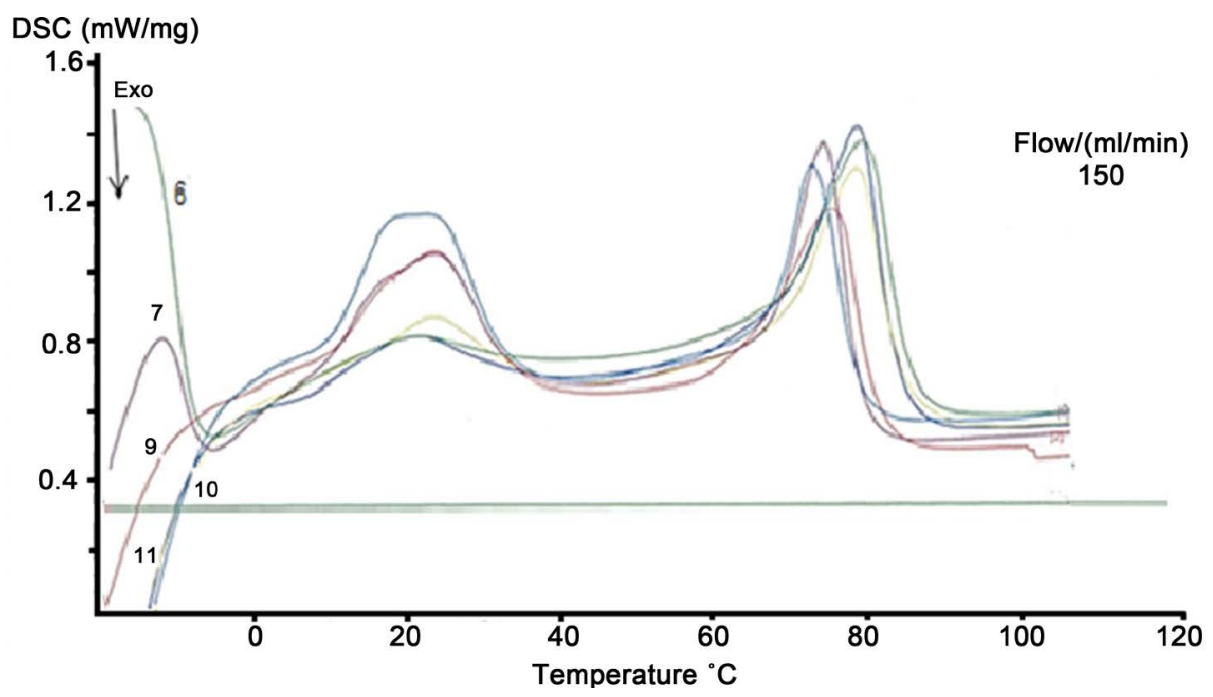

Figure 2. DSC Thermograms for the samples No's 6 - 11 as listed in Table 1.

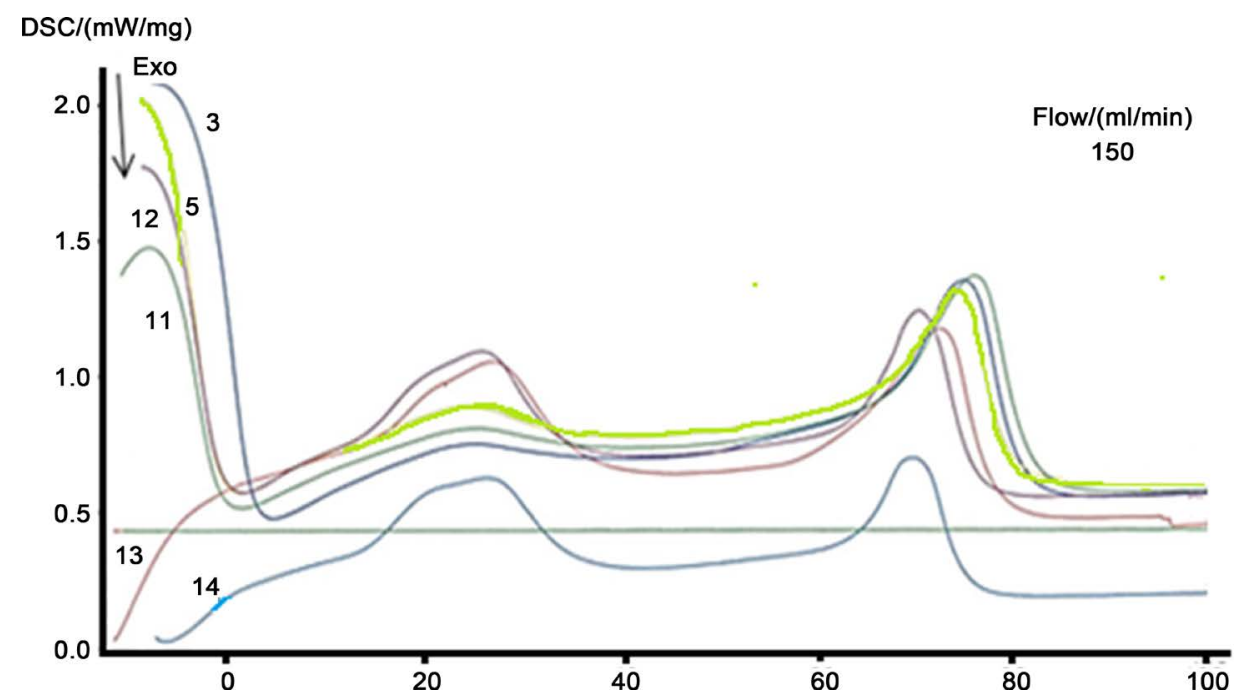

Figure 3. DSC Thermograms for the samples No's 3, 5, 11, 1, 13, 14 as listed in Table 1. 
range between $60^{\circ} \mathrm{C}-90^{\circ} \mathrm{C}$. The phase-change latent heat is calculated as the area under the curve; in the range $15^{\circ} \mathrm{C}$ to $40^{\circ} \mathrm{C}$ for pure paraffin and in the ranges $60^{\circ} \mathrm{C}$ to $90^{\circ} \mathrm{C}$ for the entire compound. As shown in the figures, during the melting and solidification process, the melting temperature $\left(\mathrm{T}_{\mathrm{m}}\right)$ for the compound decreases from $76 .{ }^{\circ} \mathrm{C}$ to $66.68^{\circ} \mathrm{C}$. The onset temperature $\left(\mathrm{T}_{\mathrm{o}}\right)$ was $54.0^{\circ} \mathrm{C}$ and $60.64^{\circ} \mathrm{C}$ respectively. The latent heat of phase change was calculated to be in the range between $154.2 \mathrm{~kJ} / \mathrm{g}$ for pure paraffin and respectively 71.3 for pure Elvax 265. For the compound we observed an increased $71.3 \mathrm{~kJ} / \mathrm{g}$ in the heat of fusion in sample No, 14 that contains 25\% kaolin and 25\% Paraffin. The melting point $\mathrm{T}_{\mathrm{m}}$ was approximately $49.3^{\circ} \mathrm{C}$.

With increasing kaolin content the melting peak was narrower and the fusion area in general bigger. Because the paraffin was not a pure substance, it's melting and solidification ranges were wider, compared to those of the pure substance. Furthermore, adding different ingredient as in Table 2 at varying concentrations alters the endothermic and exothermic curve. The selected ingredients increased the endothermic and exothermic peak and delayed the end of the melting point of the phase change as it's obvious in the thermo grams Figures 1-3.

To visualize the effects of paraffin concentrations on altering the thermal behavior of the composite, Figure 4 shows the Melting temperature changes versus Paraffin concentration.

\subsubsection{Melt Flow Index}

Melt flow index is an indirect measure of Molecular Weight $\left(M_{W}\right)$, whereby high melt flow rate corresponding to low $\mathrm{M}_{\mathrm{W}}$. In this paper, the melt flow index test was carried out according to ASTM standards D1238 at $190^{\circ} \mathrm{C}$ and applied load $2.160 \mathrm{Kg}$ for 10 minutes on a CEAST MFI-machine to investigate the effect of wax, and Kaolin which affects flow behavior of the composite. Further to examine the degradation effect due to cyclic heating cooling of the TES materials. That way we considered multiple extrusion tests to simulate the degradation mechanism. The melt flow index results are shown in Figure 4. The change in

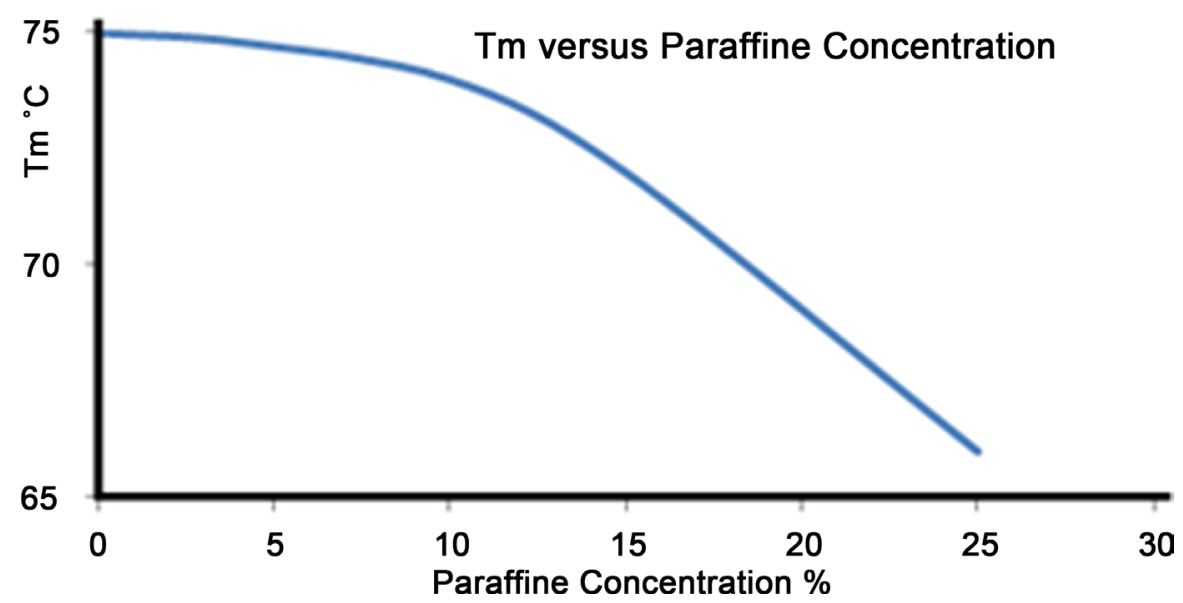

Figure 4. Melting Temperature (Tm) versus paraffin concentration. 
MFI is very clearly exponential. That was indicative for all formulations. This exponential behavior leads to the conclusion that organic materials are limited suitable to be applied to cyclic heating and cooling applications. Figure 5, multiple extrusion results for sample 14, which contains $25 \%$ kaolin and $25 \%$ paraffin. Other sample show similar behavior.

\subsubsection{FTIR}

The photoxidative decomposition due to multiple extrusion of the sample has been investigated by FTIR. FTIR spectrum for sample 14 that was subjected to multiple extrusions; (Cyclic melting and solidification) is presented in Figure 6. It was obvious that the carbonyl index at a wavelength of $1714-1740 \mathrm{~cm}^{-1}$ increase with increased extrusion pass. Different changes have been also observed in the domain $1350-1470 \mathrm{~cm}^{-1}$. It is well known that absorption bands around $1710 \mathrm{~cm}^{-1}$ corresponding to degradation and scissoring vibration of the methylene group. Scission of the methyl group in the polymer chains lead to degradation degradation, that in return changes its thermal and mechanical properties.

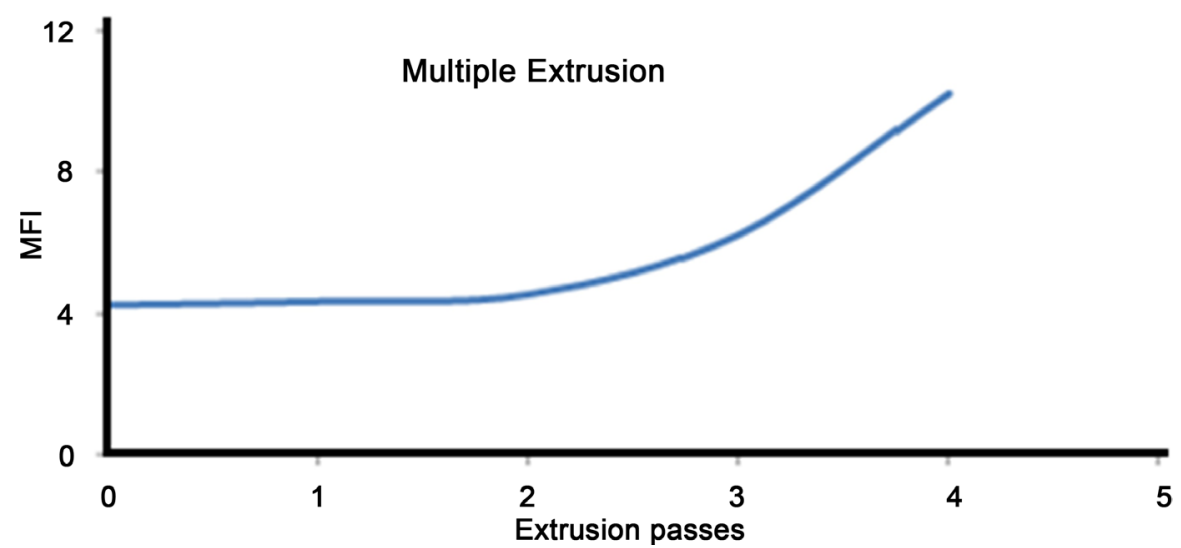

Figure 5. MFI versus No. of extrusion passes for sample No. 14.

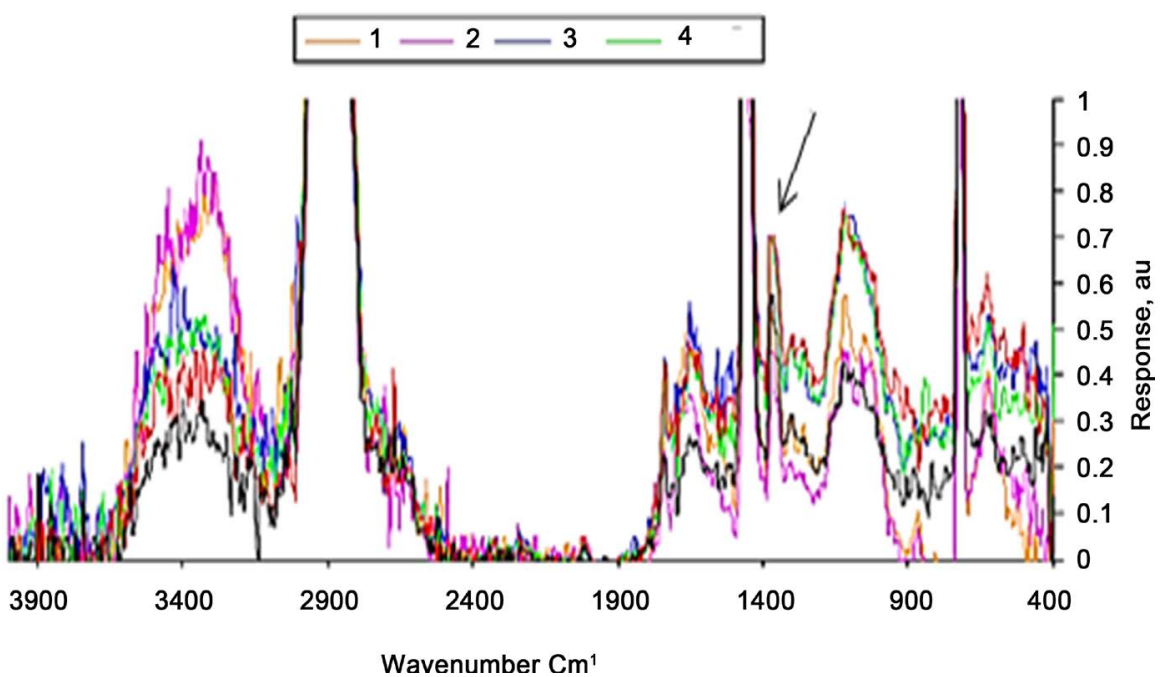

Figure 6. Is an example of the degradation effect that takes place for TES material during melting and solidification. 


\section{Results and Discussion}

The possibility to use PCMs in engineering applications is related to their capability to store/release thermal energy in a useful temperature interval. Therefore, the investigation of the thermal properties of the prepared specimens plays a major role in the present investigation. DSC thermograms of the paraffin blend and of the relative Nano composites during the first heating scans are shown in the figures.

Figures 1-3 shows the DSC plots of the prepared composite. Table 3 gives the data of the endothermic phase change temperature and heat during heating. For a better overview of the plots, only the heating thermograms are shown, whereby the cooling thermograms were eliminated. Solidification and crystallization peaks are opposite to melting. In Figures 1-3 the melting peak at low temperature range is associated with melting of the paraffin, while the second endothermic signal in the range $60^{\circ} \mathrm{C}-80^{\circ} \mathrm{C}$ is related to the melting of the polyethylene Elvax composite, for pure Elvax (reference sample. the melting point was as $78.5^{\circ} \mathrm{C}$. which is in line with manufacturer data. Adding Kaolin and paraffin change the melting and crystallization temperature.

In all plots, the melting peak of the paraffin stars at low temperature $14^{\circ} \mathrm{C}$ and the peak ends at around $30^{\circ} \mathrm{C}$ due to the presence of the different concentration of the additive. As expected, the Melting point was influenced by the additives added. It was observed the change in melting temperature as presented in Figure 4 is due to the presence of the paraffin since kaolin does not melt in this range. It is assumed that the penetration of additives in the microstructure changes the melting behavior of pure Polyethylene. The intensity of the peak associated to the melting of the composite is higher than that of the paraffin. Pure paraffin shows a broader and higher melting enthalpy values (Sample no 14 in Figure 3), compare to those compounds dedicated to the PCM. A similar trend can be detected in the cooling stage; the crystallization peak of the paraffin is located at $10^{\circ} \mathrm{C}$, while that of Elvax 265 is around $68^{\circ} \mathrm{C}$ (not reported for the sake of brevity). Samples contain high dosages of kaolin (25\%) increases the heat of fusion almost twice of that pure Elvax 265. It confirms our expectations that kaolin absorbs energy and contributes to increase heat of fusion.

The most important results from the DSC thermograms are summarized in Table 3.

Cyclic heating and freezing of PCM organic material will cause degradation. Multiple extrusions are a method associated with the thermal degradation of organic materials. This degradation behavior changes the Molecular weight and Crystallinity (also the associated $\Delta \mathrm{H}$ ). As a result the thermal degradation; melt flow index increased exponential with extrusion passes. This behavior possesses the assumption that organic material; especially Polyethylene materials and compounds of their off are not the best choice for PCM.

\section{Conclusions}

The present study is an experimental investigation to enhance thermal transition of prepared Nano composite (PCMs) contains paraffin and Nano kaolin par- 
ticles with CNT nanoparticles. Different characterization techniques were applied to determine phase change behavior, such as the supercoiling degree, phase change temperature, latent heat, and thermal stability.

Results demonstrate that addition of Nano kaolin particles in combination with thermoplastic Polyethylene (PE) materials increase the absorbed or release energy twice as of the pure Polyethylene. Increase heat of fusion is significant for our work and is in line with our expectations. Enthalpy increase was targeted because it tells us how much heat (energy) is stored or lost in the system. Any work related to develop TES materials has to consider an increase in enthalpy of the developed compound.

The thermal transition of the Nano composite PCMs is significantly enhanced by adding CNN's.

Organic PCMs containing kaolin presented acceptable thermal reliability, chemical stability, and heat transfer characteristics, thereby reflecting its acceptability for low-temperature solar thermal energy storage applications.

\section{Acknowledgements}

This work was carried out during Sabbatical leave granted to the author Prof. Amin Al Rolaid from Al Balqa' Applied University (BAU) during the academic Year 2017/2018.

\section{References}

[1] Zalba, B., Marin, J.M., Cabeza, L.F. and Mehling, H. (2003) Review on Thermal Energy Storage with Phase Change: Materials, Heat Transfer Analysis and Applications. Applied Thermal Engineering, 23, 251. https://doi.org/10.1016/S1359-4311(02)00192-8

[2] Farid, M.M., Khudhair, A.M., Razack, S.A. and Al-Hallaj, S. (2004) A Review on Phase Change Energy Storage: Materials and Applications. Energy Conversion and Management, 45, 1597. https://doi.org/10.1016/j.enconman.2003.09.015

[3] Zhang, P., Xia, L. and Wang, R.Z. (2011) The Thermal Response of Heat Storage System with Paraffin and Paraffin/Expanded Graphite Composite for Hot Water Supply. World Renewable Energy Congress-Electronic Conference Proceedings, Linköping, 8-13 May 2011, 57. https://doi.org/10.3384/ecp11057756

[4] Baylin, F. (1979) Low Temperature Thermal Energy Storage: A State of the Art Survey. Report No. SERI/RR/-54-164. Solar Energy Research Institute, Golden.

[5] Dorigato, A., Canclini, P., Unterberger, S.H. and Pegoretti, A. (2017) Phase Changing Nanocomposites for Low Temperature Thermal Energy Storage and Release. eXPRESS Polymer Letters, 11, 738-752. https://doi.org/10.3144/expresspolymlett.2017.71

[6] Shankar Narayanan, S. (2017) Development of Sunlight-Driven Eutectic Phase Change Material Nanocomposite for Applications in Solar Water Heating. Resource-Efficient Technologies, 3, 272-279.

[7] Fredi, G. and Dorigato, A. (2017) Wax Confinement with Carbon Nanotubes for Phase Changing Epoxy Blends. Polymers, 9, 405. https://doi.org/10.3390/polym9090405

[8] Al Rolaid, A., Anagreha, N., A-L Addousb, M. and Massadeha, S. (2013) Crystalli- 
zation Behavior of iPP/LLDPE Blend Filled with Nano Kaolin Particles. Jordan Journal of Mechanical and Industrial Engineering, 7, 35-39.

[9] Al Rolaid, A. (2009) Synergistic Effect of TNPP and Carbon Black in Weathered XLPE Low and Medium Voltage Cable. European Polymer (EPF09) Conference, Graz, July 2009.

[10] Kosny, J., Shukla, N. and Fallahi, A. (2013) Report; Energy Efficiency and Renewable Energy. US Departement of Energy, Washington DC.

[11] Lingayat, A.B. and Suple, Y.R. (2013) A Review on Phase Change Material in Thermal Energy Storage Medium. International Journal of Engineering Research and Applications (IJERA), 3, 916-921.

[12] Kohl, M., Meir, M.G., Papillon, P., Wallner, G.M. and Saile, S. (2012) Polymeric Materials for Solar Thermal Applications, First Edition. Wiley-VCH Verlag GmbH \& Co. KGaA, Weinheim. https://doi.org/10.1002/9783527659609

[13] Zafer UREM. Sc., C. Eng., MCIBSE, MASHRAE, M.Inst.R (2011) MIIR Phase Change Material Products Limited Published in the Symposia. Cibse Technical Symposium-2011, Leicester, 6-7 September 2011.

[14] Chen, J.Q., Yang, D.H. and Jiang, J.H. (2014) Research Progress of Phase Change Materials (PCMs) Embedded with Metal Foam (A Review). Procedia Material Science, 4, 389-394. https://doi.org/10.1016/j.mspro.2014.07.579

[15] Chen, L., Zou, R., Xia, W., Liu, Z., Shang, Y., Zhu, J., Wang, Y., Lin, J., Xia, D. and Cao, A. (2012) Electro- and Photodriven Phase Change Composites Based on Wax-Infiltrated Carbon Nanotube Sponges. ACS NANO, 6, 10884-10892. https://doi.org/10.1021/nn304310n

[16] Shaikh, S., Lafdi, K. and Hallinan, K. (2008) Carbon Nano Additives to Enhance Latent Energy Storage of Phase Change Materials. Journal of Applied Physics, 103, Article ID: 094302. https://doi.org/10.1063/1.2903538

[17] Tabassum, H., Huang, X., Chen, R. and Zou, R. (2015) Tailoring Thermal Properties via Synergistic Effect in a Multifunctional Phase Change Composite Based on Methyl Stearate. Journal of Materiomics, 1, 229-235.

[18] Huang, J. (2013) Form-Stable Phase Change Materials Based on Eutectic Mixture of Tetradecanol and Fatty Acids for Building Energy Storage: Preparation and Performance Analysis. Materials, 6, 4758-4775.

[19] Al Rolaid, A. (2013) Development of Novel Polymer Phase Change Material for Heat Storage Application. International Journal of Material Science and Application, 2, 168-172.

[20] Tiat, V.H.K. and Barrio, E.P. (2011) Recent Patents on Phase Change Materials and Systems for Latent Heat Thermal Energy Storage. Recent Patents on Mechanical Engineering, 4, 16-28. https://doi.org/10.2174/2212797611104010016

[21] Martinot, E., Dienst, C., Liu, W. and Chai, Q. (2007) Renewable Energy Futures: Targets, Scenarios, and Pathways. Annual Review of Environment and Resources, 32, 205-239.

[22] Joint EASE/EERA Recommendations for a European Energy Storage Technology Development Roadmap towards 2030.

[23] Report Status and Recommendations for RD\&D on Energy Storage Technologies in a Danish Context. 